\title{
Le système éducatif de la Tanzanie continentale
}

Nathalie Bonini et Suleman Sumra

\section{OpenEdition}

Journals

Édition électronique

URL : https://journals.openedition.org/ries/2654

DOI : 10.4000/ries.2654

ISSN : 2261-4265

Éditeur

France Education international

Édition imprimée

Date de publication : 1 décembre 2012

Pagination : 18-25

ISSN : 1254-4590

Référence électronique

Nathalie Bonini et Suleman Sumra, «Le système éducatif de la Tanzanie continentale », Revue internationale d'éducation de Sèvres [En ligne], 61 | décembre 2012, mis en ligne le 06 février 2015, consulté le 06 juillet 2021. URL : http://journals.openedition.org/ries/2654 ; DOI : https://doi.org/ $10.4000 /$ ries.2654

Ce document a été généré automatiquement le 6 juillet 2021

(c) Tous droits réservés 


\title{
Le système éducatif de la Tanzanie continentale
}

\author{
Nathalie Bonini et Suleman Sumra
}

1 La République unie de Tanzanie est née en 1964, de l'union politique entre le Tanganyika et l'archipel de Zanzibar situé à une trentaine de kilomètres au large de la côte est. Elle compte environ quarante millions d'habitants avec un taux d'alphabétisation s'élevant à un peu moins de $70 \%$. Zanzibar dispose de son propre gouvernement et de son propre ministère de l'éducation, qui lui confèrent ainsi une certaine autonomie dans l'organisation et la gestion de son système éducatif. Le paysage scolaire (notamment marqué par une importante différenciation de l'offre scolaire), la structure de l'éducation et les orientations éducatives de l'archipel et du continent différant sensiblement, ce panorama du système éducatif tanzanien sera limité à la partie continentale du pays. Le PIB par habitant de Tanzanie figure parmi les plus bas du monde et l'économie dépend fortement de l'agriculture qui fournit $85 \%$ des exportations et emploie $80 \%$ de la population active. L'exploitation des ressources naturelles et surtout le tourisme (notamment dans les parcs nationaux) représentent également des postes importants de l'économie. Selon les récents rapports nationaux du ministère de l'Éducation nationale de Tanzanie ${ }^{1}$, les dépenses publiques d'éducation ${ }^{2}$ représentaient, pour l'année 2009/2010, 18,3 \% du budget de l'État alors qu'elles avaient culminé à 27,4 \% en 2008, le taux le plus bas ayant été de 13,3\% en 1985.

\section{Administration et structure du système éducatif}

\section{L'administration de l'éducation}

2 L'éducation relève en Tanzanie de la responsabilité du ministère de l'Éducation et de la formation technique (Ministry of Education and Vocational Training, MoEVT). Il administre l'éducation de base qui comprend l'enseignement pré-primaire, le primaire, le secondaire, le supérieur, la formation des adultes et l'éducation non formelle, la formation et la gestion du personnel enseignant ainsi que l'enseignement technique. 
Toutefois, les administrations régionales (dirigées par un Regional Education Officer) et locales (sous la houlette du District Education Officer) ont la responsabilité de la gestion ordinaire des écoles primaires (qui abritent également les classes de pré-primaires) et secondaires, tandis que le MoEVT décide des politiques éducatives, des programmes et est garant de la qualité et du bon fonctionnement de cet enseignement de base. Les établissements privés du cycle primaire comme du secondaire sont gérés par leurs propriétaires en conformité avec les termes de référence du MoEVT. L'inspection a le pouvoir de contrôler toute école, privée ou publique, même si les inspecteurs restreignent le plus souvent leurs visites aux établissements secondaires et de formation des enseignants (Teacher Colleges: TCs). Le conseil d'accréditation, Higher Education Accréditation Council (HEAC), coordonne les différents niveaux d'éducation et contrôle la qualité de l'éducation.

3 L'une des particularités du système éducatif tanzanien est son bilinguisme, kiswahili (langue bantoue) et anglais. Le kiswahili constitue la langue d'instruction dans l'enseignement primaire, tandis que l'anglais, qui est enseigné comme langue étrangère dès la troisième année du primaire, devient la langue d'instruction à partir du niveau secondaire; affecté d'un coefficient important aux examens de cycle secondaire, le kiswahili continue d'être enseigné en tant que matière obligatoire.

4 Jusqu'à l'abolition du parti unique et la vague de libéralisation qui l'a accompagné au milieu des années 1990, l'État tanzanien a maintenu un strict contrôle sur l'éducation, ne laissant qu'une opportunité très réduite aux initiatives d'éducation privées. Les écoles non gouvernementales, qui avaient timidement commencé à s'implanter dix ans plus tôt, se développent ainsi à partir de cette époque. Elles concernent essentiellement les niveaux secondaires; le pays ne compte aujourd'hui encore que très peu d'institutions primaires privées.

5 Pour atteindre les objectifs fixés en 2000 lors du Forum de l'éducation pour tous (EPT) à Dakar, le gouvernement tanzanien a adopté une série de réformes qui ont à la fois accru le taux de scolarisation et modifié le paysage éducatif. En 1995 est lancée l'Education and Training Policy. Cette réforme inaugure une série de plans définissant les orientations éducatives principales et, suivant une politique globale de décentralisation qui s'organise à l'aube des années 2000 , confie la gestion et l'administration de l'éducation - d'abord primaire puis secondaire - aux niveaux local et régional. Désormais, le gouvernement central prend en charge le paiement des salaires des enseignants et les équipements scolaires, tandis que les administrations locales (de district et de canton) ont la responsabilité de la construction et de la maintenance des écoles ainsi que de leurs dépenses de fonctionnement et de gestion. Les familles doivent, elles, supporter le coût du matériel pédagogique de base, de l'uniforme et des frais annexes.

\section{L'éducation de base}

6 Jusqu'à la mise en place du Primary Education Development Plan (PEDP-I 2002-2006) en 2002, l'enseignement pré-primaire (5-6 ans), qui n'est pas obligatoire en Tanzanie, n'était pas (ou très partiellement) pris en charge par le gouvernement; les quelques structures existantes (surtout en ville) étaient gérées par des acteurs privés (ONG, institutions religieuses ou autres). Depuis, ce niveau d'enseignement se développe rapidement, y compris dans le secteur public, les écoles primaires ayant désormais l'injonction de créer des classes d'enseignement pré-primaire. Toutefois, du fait du 
manque de place et d'enseignants - pris sur le contingent de ceux du primaire -, toutes les écoles ne possèdent pas ces petites classes.

7 L'enseignement primaire, qui est obligatoire, se compose de sept niveaux, désignés par le terme anglais de "standards" et concerne théoriquement les enfants âgés de 7 à 13 ans. Depuis 2002, les frais de scolarité, qui avaient été rétablis en 1994 après vingt années de gratuité, ont de nouveau ont été abolis en même temps que l'obligation de scolarisation à ce niveau était réaffirmée. Depuis la mise en œuvre de la deuxième phase du plan de développement de l'école primaire (PEPD II 2007-2011), le curriculum des écoles primaires comprend douze matières, à savoir les mathématiques, les sciences, l'histoire, la géographie, l'instruction civique, le kiswahili, l'anglais, les nouvelles technologies de l'information, la méthodologie de travail, les sports et loisirs, le français et la religion ${ }^{3}$. Les élèves peuvent redoubler du standard 1 à 4 mais n'y sont théoriquement plus autorisés au-delà, sauf cas exceptionnel. Si, comme on l'a noté, la langue d'enseignement dans les écoles primaires est le kiswahili, quelques rares écoles privées sont aujourd'hui autorisées à utiliser l'anglais. Le cycle primaire se clôt par un examen national, le Primary School Leaving Examination (PSLE), qui permet à ceux qui le réussissent d'accéder à l'enseignement secondaire public. En cas d'échec, les élèves ne sont en principe pas autorisés à le tenter à nouveau mais peuvent en revanche intégrer des écoles secondaires privées, qui seules accueillent les non diplômés, ou suivre un enseignement technique post-primaire de deux années dans des centres professionnels (vocational schools). Le comité national de sélection (NECTA) examine les dossiers des élèves reçus et, en fonction de leurs notes, les affecte dans les différentes écoles gouvernementales, les plus prestigieuses étant en principe réservées aux meilleurs élèves.

8 L'enseignement secondaire est divisé en deux cycles: le cycle inférieur (lower secondary), d'une durée de quatre ans (de form 1 à form 4) et le cycle supérieur, (upper secondary), de deux ans (forms 5 et 6). Le passage de l'un à l'autre est conditionné au résultat de l'examen national à la fin de la quatrième année (form 4$)$ : le Certificate of Secondary Education Examination (CSEE), équivalent du O'Level britannique. Si les élèves le réussissent en obtenant une note moyenne de 1 à 3 (division I to III) ${ }^{4}$, ils peuvent intégrer le cycle secondaire supérieur. Ce second cycle se termine lui aussi par un examen: Advanced Certificate of Secondary Education (ACSE), équivalent du A-level britannique, qui permet la poursuite d'études à l'université ou dans d'autres institutions supérieures. Le curriculum des écoles secondaires est composé d'enseignements de base et obligatoires et d'enseignements optionnels, toutes les écoles ne proposant pas les mêmes options. Pour obtenir le certificat de fin de premier cycle du secondaire (CSEE), les étudiants doivent choisir au moins sept matières, dont les mathématiques, l'anglais, le kiswahili, la biologie et l'instruction civique, qui sont obligatoires.

$9 \quad$ Les écoles secondaires publiques sont de deux types : les écoles à rayonnement national ou régional et les écoles communautaires 5 . Plus récentes, ces dernières sont initiées, construites et, pour une large part, financées par les habitants d'une circonscription. Moins prestigieuses, elles ont un recrutement essentiellement local et n'offrent pour la plupart que les quatre premières années d'enseignement. Elles constituent la très grande majorité des écoles secondaires ouvertes depuis les années 2000 mais leur implantation varie considérablement d'une région à l'autre. Malgré l'essor des écoles privées à partir des années 1990, elles sont aujourd'hui moins nombreuses que 
les écoles secondaires publiques mais proportionnellement plus nombreuses à proposer un enseignement en forms 5 et 6 (upper secondary) ${ }^{6}$.

Le secteur de l'éducation non formelle se compose principalement de l'alphabétisation des adultes, mise en œuvre dans le cadre du Community Based Adult Education (ICBAE) et de programmes d'éducation « de seconde chance » ciblant les jeunes de 11 à 18 ans hors système scolaire dans les centres d'apprentissage COBET (Complementary Basic Education in Tanzania). Ce dispositif destiné aux jeunes gens qui n'ont pas été scolarisés ou qui ont, pour diverses raisons, abandonné l'école, fut instauré à la fin des années 1990 ; il leur permet, à terme, d'intégrer le système formel d'éducation à différents niveaux ${ }^{7}$.

\section{Évolution du paysage scolaire tanzanien}

\section{Le niveau primaire}

11 Jusqu'à très récemment, le gouvernement a essentiellement centré ses efforts sur le développement de l'éducation primaire; à la fin des années 1970, la scolarisation primaire universelle avait pratiquement été atteinte avant que le taux de scolarisation ne chute pendant les deux décennies suivantes marquées par une sévère crise économique et la mise en place de plans d'ajustement structurel qui ont notamment engendré une forte réduction des dépenses publiques. Si l'ensemble du secteur de l'éducation en fut affecté, la baisse du taux de scolarisation en primaire fut à la mesure de sa forte progression antérieure. La tendance s'est ensuite inversée à l'aube des années 2000, notamment après l'instauration du PEDP-I et de l'une de ses mesures phares : l'abolition des frais de scolarité (tableau 1).

Tableau 1

Taux de scolarisation dans l'enseignement primaire en Tanzanie

\begin{tabular}{|l|c|c|c|c|c|}
\hline Années & 1981 & 1990 & 2000 & 2006 & 2010 \\
\hline Taux nets de scolarisation & $68 \%$ & $55 \%$ & $59 \%$ & $96 \%$ & $95 \%$ \\
\hline Taux bruts de scolarisation & $94 \%$ & $74 \%$ & $78 \%$ & $118 \%$ & $106 \%$ \\
\hline
\end{tabular}

Sources : arrondis au nombre entier le plus proche, les taux de scolarisation ci-dessus proviennent de différentes sources : MoEVT (2010) ; Unesco-IBE (2010) ; URT (2008).

L'expansion de la scolarisation n'a pas été uniforme sur tout le territoire et, en dépit de la volonté de développer l'offre scolaire dans les zones les moins bien dotées condition sine qua non pour atteindre l'objectif de scolarisation universelle -, le fossé entre les régions les plus riches, généralement les plus urbanisées et qui affichent de forts taux de scolarisation et les régions les plus pauvres, comprenant surtout des zones rurales à faible densité de population, moins scolarisées, n'a pas été comblé. À titre d'exemple, la région du Kilimanjaro, dont la supériorité en matière d'éducation scolaire remonte aux toutes premières heures de la colonisation via l'implantation de nombreuses écoles missionnaires, possédait en 2003 plus du double des écoles de n'importe quelle autre région et trois fois plus que la plupart d'entre elles ${ }^{8}$.

\section{La croissance de l'enseignement secondaire}

13 Un élément frappant de la situation éducative en Tanzanie est la faiblesse de l'éducation secondaire comparée à l'éducation primaire, même si l'écart s'est 
largement restreint ces toutes dernières années. Après en avoir bridé l'expansion, le gouvernement tanzanien a opté pour une politique d'expansion de l'enseignement secondaire au milieu des années 1980. Le véritable essor du second cycle date de l'instauration en 2004 du Secondary Education Development Plan (SEDP), organisé en trois phases de développement de cinq ans chacune. Ce plan a conduit à la construction de plus d'un millier d'écoles secondaires gouvernementales, essentiellement communautaires, entre 2003 et 2006 ; progression qui s'est accentuée les années suivantes, le nombre d'écoles secondaires gouvernementales passant de 829 en 2004 à 3397 en 2010, tandis que, dans la même période, le nombre d'écoles secondaires privées passait de 462 à $869^{9}$. L'immense majorité de ces écoles secondaires sont des écoles communautaires offrant, comme on l'a noté, un enseignement pour les quatre premières années du cursus uniquement.

Cette augmentation de la capacité d'accueil des écoles secondaires, combinée à l'amélioration du taux de réussite à l'examen de fin de primaire - la proportion d'élèves ayant réussi l'examen de fin de primaire est passée de $27,1 \%$ en 2002 à $61,8 \%$ en 2005, a contribué à la très forte augmentation des effectifs, notamment dans la première année du cycle (form 1). En 1995, 15 \% des élèves sortant du primaire étaient inscrits à l'école secondaire : ils étaient $49 \%$ dix ans plus tard. Demeuré aux alentours de $3 \%$ de 1970 au milieu des années 1980, le taux net de scolarisation dans le cycle secondaire a donc progressé pendant les deux décennies suivantes; il était de $6 \%$ en 2000, de $13 \%$ en 2006 et a atteint près de $28 \%$ en 2009. Notons toutefois que ce taux de scolarisation regroupe les taux nets de scolarisation des deux cycles du secondaire, qui sont très différents selon qu'ils concernent les quatre premières années (lower secondary) ou les deux dernières (upper secondary). En effet, les déperditions très importantes entre ces deux niveaux résultent à la fois des nombreux abandons pendant les trois années du premier cycle du secondaire ${ }^{10}$, du taux de réussite à l'examen du CSEE qui, bien qu'en nette augmentation depuis les années 2000 (il était en 1999 de 21,3\%) demeure sous la barre des $40 \%$ en 2009, et d'une faiblesse de l'offre scolaire pour les deux dernières années du cycle secondaire. En 2010, seuls 524 établissements comprenaient les six années du cycle secondaire, menant les élèves jusqu'au A-level, dont 365 privés et 179 publics $^{11}$.

15 La rapide expansion de l'enseignement secondaire a ses revers en terme de qualité, le principal étant la pénurie de professeurs, et notamment de professeurs formés et compétents pour enseigner à ce niveau. De plus, cette forte croissance engendre autant qu'elle les masque les carences matérielles de locaux, de fournitures scolaires, etc., qui sont particulièrement criantes en milieu rural où est concentrée de surcroît la grande majorité des personnes pauvres du pays. Enfin, si la parité entre les garçons et les filles est effective dans l'enseignement primaire, ces dernières sont moins nombreuses que leurs homologues sur les bancs du secondaire et encore moins présentes dans le cycle supérieur.

\section{Les études supérieures et la formation des enseignants}

16 Les élèves qui ont passé avec succès le dernier examen national à la fin de secondaire (ACSE) peuvent intégrer l'université ou d'autres établissements supérieurs privés ou publics. Contrairement à l'éducation de base qui est gérée séparément par les ministères du continent et de Zanzibar, l'éducation supérieure est du ressort de l'union. 
L'éducation supérieure se divise en deux branches : l'université et les institutions non universitaires proposant un enseignement de trois ans post--secondaire menant à un diplôme. L'université peut dispenser des diplômes au-delà des trois années nécessaires pour obtenir une licence (Bachelor degree). L'université de Dar es Salaam (UDSM), qui était une branche de l'université d'Afrique de l'Est, est devenue indépendante et sous le contrôle de l'État en 1970. En 1999 a été mis en place un programme de développement de l'enseignement supérieur, la Higher Education Policy. Selon un rapport de $2010^{12}$, entre 1997 et 1999, les inscriptions d'étudiants en première année ont augmenté de $50 \%$ après des années de stagnation dues à la faiblesse de l'offre scolaire supérieure mais surtout à celle du taux de scolarisation secondaire pendant de nombreuses années. Le nombre total d'étudiants pour l'année universitaire 2006/2007 était de près de cinquante mille, dont $78 \%$ dans les universités publiques et $21,5 \%$ dans les universités privées $^{13}$. On comptait en 2008 quarante-trois établissements, dont vingt-et-une universités ${ }^{14}$ (huit publiques et treize privées), quatre instituts supérieurs techniques et dix-huit institutions et collèges agréés par le ministère.

Initialement, la certification des enseignants était composée de trois niveaux: le grade C (le plus faible), le grade B et le grade A, les deux premiers concernant des enseignants ayant réussi l'examen de fin de primaire (PLSE) ou ayant poursuivi quelques années d'études dans le secondaire, tandis que le grade A s'adressait aux lauréats de l'examen national CSEE (form 4). Depuis quelques années, le gouvernement tanzanien a réformé la formation des enseignants dans les instituts de formation des maîtres pour les amener à l'équivalent du grade $\mathrm{A}$, soit par un programme de formation de deux ans destiné aux élèves sortant du premier cycle du secondaire, soit par un programme de formation interne, In-Service programme, mis en place à la fin des années 1990 pour les enseignants du primaire déjà en poste qui ne disposent pour tout bagage scolaire que du certificat de fin d'études primaires. De nos jours, la formation des enseignants peut ainsi être dispensée soit par les Teacher Colleges, soit par l'université. Les enseignants formés dans les TCs reçoivent un enseignement académique et professionnel d'une durée de deux ans et sont amenés à enseigner aux niveaux pré-primaire, primaire et dans le premier cycle du secondaire (en fonction de leur niveau scolaire à l'entrée au Teacher College). La formation à l'université leur permet de préparer une licence spécialité éducation, à l'issue de laquelle, munis du diplôme (diploma), ils peuvent enseigner dans le cycle secondaire supérieur ou dans les Teacher Colleges eux-mêmes. Cette formation, qui était de quatre ans avant 2005, a été réduite à trois ans pour pallier le manque d'enseignants qu'a engendré la forte augmentation de la scolarisation secondaire.

\begin{tabular}{|l|l|}
\hline $\begin{array}{l}\text { Quelques données } \\
\text { sur la République unie de Tanzanie }\end{array}$ \\
\hline Superficie & $945087 \mathrm{~km}^{2}$ \\
\hline Population & $\begin{array}{l}46218 \text { millions } \\
\text { (estimation 2011) }\end{array}$ \\
\hline Capitale & $\begin{array}{l}\text { Dodoma } \\
\text { (capitale officielle, } \\
\text { siège du Parlement) }\end{array}$ \\
\hline
\end{tabular}




\begin{tabular}{|l|l|}
\hline Capitale économique & Dar es Salaam \\
\hline Langues officielles & Kiwahili, anglais \\
\hline $\begin{array}{l}\text { Croissance } \\
\text { démographique }\end{array}$ & $2,9 \%$ \\
\hline Taux d'alphabétisation & $70 \%$ \\
\hline $\begin{array}{l}\text { Indice } \\
\text { de développement humain }\end{array}$ & $\begin{array}{l}159^{\mathrm{e}} \text { rang sur } 177 \\
(2008)\end{array}$ \\
\hline PIB par habitant & $525,6 \$(2009)$ \\
\hline
\end{tabular}

Source : Banque mondiale et UN-Data : http://data.un.org/

\section{BIBLIOGRAPHIE}

Ministry of Education and Vocational Training (MoEVT) (2010): Basic Education Statistics in Tanzania, http://educationstatistics.moe.go.tz/moe/.

National Bureau of Statistics (NBS) [Tanzania] \& ORC Macro, Tanzania Demographic and Health Survey (DHS) 2004-05, octobre 2005, Dar es Salaam, Tanzania, 381 p.

Tanzania : ICT in Education Situational Analysis, juillet 2010, http://www.gesci.org/assets/files/ Knowledge\%20Centre/Situational\%20Analysis_Tanzania.pdf/.

Unesco-IBE, Données mondiales de l'éducation, $7^{e}$ édition, 2010-2011, http://

www.ibe.unesco.org/fileadmin/user_upload/Publications/WDE/2010/pdf-versions/

United_Republic_of_Tanzania.pdf/.

United Republic of Tanzania. The development of Education. National Report of United Republic of Tanzania, octobre 2008, http://www.ibe.unesco.org/National_Reports/ICE_2008/ tanzania_NR08.pdf/.

\section{NOTES}

1. Sources: United Republic of Tanzania. National Report of United Republic of Tanzania, The development of Education, octobre 2008, http://www.ibe.unesco.org/National_Reports/ICE_2008/ tanzania_NR08.pdf/; Ministry of Education and Vocational Training (2010) : Basic Education Statistics in -Tanzania, http://educationstatistics.moe.go.tz/moe/; Tanzania: ICT in Education Situational Analysis, -juillet 2010, http://www.gesci.org/assets/files/Knowledge\%20Centre/ Situational\%20Analysis_Tanzania.pdf/ 
2. Les dépenses publiques d'éducation comprennent toutes les dépenses liées aux différents établissements scolaires et autres institutions éducatives (à la fois publics et privés), l'administration de l'éducation ainsi que diverses subventions (bourses d'études, logements, etc.). 3. Cette nouvelle phase de mise en œuvre du PEPD comporte également trois nouveaux axes de développement: l'inclusion des questions transversales (telles celles relatives au VIH, à la protection de l'environnement et à l'égalité homme/femme), une réflexion sur les pratiques éducatives et l'amélioration de la surveillance et de l'évaluation.

4. Les tests de contrôle continu et examens sont notés de 1 à 4 (division I to division IV), 4 étant la note la plus basse.

5. Voir Bonini N., «Les écoles communautaires dans les zones rurales de Tanzanie », Revue internationale d'éducation de Sèvres, ${ }^{\circ}{ }^{59}$, avril 2012, 83-92.

6. En juin 2010, il y avait en Tanzanie 4266 écoles secondaires dont 3397 écoles publiques et 869 privées. 3742 écoles, dont 520 privées (soit $14 \%$ ) et 3218 publiques ( $86 \%$ ) offraient un enseignement de premier cycle (lower secondary). Parmi ces 3218 écoles publiques, 3216 sont des écoles communautaires. 524 établissements comprenaient les six années du cycle secondaire, menant les élèves jusqu'au ACSE, dont 365 privées (soit $65 \%$ ) et 179 publiques, dont 93 écoles communautaires, (soit 34,2 \%). Source : MoEVT, 2010, p. 72.

7. Selon le rapport national sur le développement de l'éducation, plus d'un demi-million d'enfants hors école ont pu intégrer l'école primaire via le dispositif COBET (URT, 2008, p. 12).

8. En 2004, la proportion d'hommes et de femmes n'ayant jamais été scolarisés était respectivement de $12 \%$ et de $14 \%$ dans la région de Dar es Salaam et de $12 \%$ et $15 \%$ dans celle du Kilimanjaro, contre $44 \%$ et $55 \%$ dans la région de Tabora, au centre-ouest du pays (NBS, 2005).

9. MoEVT, 2010.

10. Des études réalisées sur la cohorte d'élèves entrés en première année de cycle secondaire en 2000 révèlent que moins de $72 \%$ d'entre eux sont présents en dernière année, quatre ans plus tard (URT, 2004).

11. MoEVT, 2010.

12. Tanzania : ICT in Education Situational Analysis, 2010.

13. Ibid., p. 54.

14. Toutes les universités sont régies par la commission tanzanienne des universités (Tanzania Commission of Universities) (TCU).

\section{INDEX}

Palabras claves : bilingüismo, educación básica, enseñanza primaria, enseñanza superior, formación de docentes, sistema educativo

Keywords : bilingualism, basic education, primary education, higher education, teacher education, educational system

Mots-clés : bilinguisme, éducation de base, enseignement primaire, enseignement supérieur, formation des enseignants, système éducatif

Index géographique : Tanzanie 


\section{AUTEURS}

\section{NATHALIE BONINI}

Maître de conférences en anthropologie à l'Université François-Rabelais de Tours (UMR 6173 CITERES). Ses travaux de recherche en Tanzanie portent sur l'éducation scolaire et non scolaire des enfants, en particulier chez les pasteurs massaï, ainsi que sur le développement récent de l'éducation secondaire dans ce pays. Elle a participé à l'élaboration d'un projet ANR sur l'éducation secondaire dans cinq pays africains. Parallèlement, elle mène des recherches sur les populations récemment installées dans les Cévennes (les « néo-ruraux »), et s'intéresse en particulier à leurs pratiques d'éducation et de socialisation.

\section{SULEMAN SUMRA}

Après son doctorat à l'Université de Stanford (États-Unis), Suleman Sumra a enseigné plus de trente ans à l'Université de Dar es-Salaam, où il a été notamment vice-doyen de la recherche et des publications et où il a créé et dirigé le Bureau de la recherche et de l'évaluation jusqu'en 1997. Il a également été coordonné pour la Tanzanie un projet de recherche conjoint entre l'Université de Dar es-Salaam, l'Université de Western Cape (Afrique du Sud) et de l'Université d'Oslo (Norvège) sur la langue d'enseignement en Tanzanie et en Afrique du Sud. Consultant pour diverses organisations, il a écrit de nombreux articles sur l'enseignement primaire en Tanzanie. 